\title{
Exploring Urban Dynamics from Bluetooth Tracking Data: A Case Study of Austin, Texas
}

\author{
Yihong Yuan*, David Mills \\ Department of Geography, Texas State University, San Marcos, TX 78666, yuan@txstate.edu, dam203@txstate.edu \\ * Corresponding author
}

\begin{abstract}
In recent decades, the growing availability of location-aware devices, such as Global Positioning System (GPS) receivers and smart phones, has provided new challenges and opportunities for policy makers to analyze, model, and predict human mobility patterns. However, previous studies on Bluetooth technologies have mainly focused on applying Bluetooth data to analyzing traffic and optimizing transportation networks or deploying new Bluetooth devices in civil engineering. The use of such datasets in understanding urban dynamics and real-time land use patterns is rather limited. This study develops an extendable workflow to explore urban dynamics from Bluetooth data based on a case study in Austin, Texas. We identified similar mobility patterns in different areas of Austin during various study periods, including the Memorial Day long weekend in 2016 and a national musical festival (South by Southwest). Our main goal is to prove the efficacy of this specific workflow and methodology to understand urban dynamics based on real-time Bluetooth data. The hypothesis is that Bluetooth data is sensitive to the daily patterns of human interactions and movements on the individual level, therefore it can capture detailed dynamic patterns. The proposed research also validates new concepts such as "human sensing" and "social sensing" in the field of geography and spatial sciences, which introduces new opportunities to monitor the human aspects of social life.
\end{abstract}

Keywords: Human Mobility, Time Series Analysis, Bluetooth, Big Geodata

\section{Introduction}

In recent decades, the growing availability of locationaware devices, such as Global Positioning System (GPS) receivers and smart phones, has provided new challenges and opportunities for policy makers to analyze, model, and predict human mobility patterns (Chen et al. 2016, Salganik 2018, Shi et al. 2018, Poorthuis and Zook 2017). Commonly used datasets include, but are not limited to, georeferenced mobile phone records, location-based social media, GPS floating-car data, and Bluetooth tracking data (Delafontaine et al. 2012, Yuan and Raubal 2016, Yang et al. 2018, Costa et al. 2018). Among these data sources, information collected through Bluetooth sensors are particularly effective at capturing intra-urban mobility patterns across street networks due to their high precision and sampling frequency. The sensors consist of Bluetooth probe devices that scan for other Bluetooth-enabled devices within their radio proximity and then store the data in local drives or cloud services for future use.

Previous studies on Bluetooth technologies mainly focused on applying Bluetooth data to analyzing traffic and optimizing transportation networks, or deploying new Bluetooth systems and devices in the field of civil engineering (Reed 2014, Anderson et al. 2014). In geography, the use of such Bluetooth datasets is rather limited though, especially in understanding the functionalities of urban regions and land use patterns. Therefore, this study proposes to identify outlier urban functional regions from Bluetooth data based on a case study in Austin, Texas. Our main objective is to prove the efficacy of this specific workflow and methodology to analyze urban dynamics based on Bluetooth data. The hypothesis is that Bluetooth data is more sensitive to the daily patterns of human interactions and movements on the individual level, therefore it can capture more detailed urban dynamics and outliers than traditional Land Use and Land Cover (LULC) datasets can. Because previous studies have demonstrated that various urban regions can be characterized by their activity levels at different times of day (Ahas et al. 2015, Calabrese, Ferrari and Blondel 2015), this research also adopts time series data to characterize urban dynamics.

\section{Related Work}

Previous research has shown that Bluetooth-enabled device detection from stationary sensors can be a reliable means of sampling location data in urban areas, with enabled-devices being detected $80 \%$ of the time within 100 meters of a stationary Bluetooth sensor (Araghi et al. 2015, Crawford, Watling and Connors 2018). These datasets provide a practical way for various applications in urban planning and policy making, including but not limited to the following areas:

- Detecting road traffic: Bluetooth technology has opened the possibilities for researchers to understand the mobility landscape from a new perspective. Traditionally, a network of stationary sensors have been affixed at 
locations such as traffic lights to detect traffic flow (Du et al. 2015, Silva and Moreira 2012). However, Bluetooth research methodologies are not limited to stationary sensors. Researchers also have the capability to deploy sensors into moving vehicles for capturing an improved understanding of the mobility landscape (Filgueiras et al. 2014, Friesen and McLeod 2015, Araghi et al. 2015).

- Identifying urban land use: Policy makers and community planners may benefit from the availability of pedestrian movement data provided by Bluetooth sensors when analyzing urban land use patterns (Malinovskiy, Saunier and Wang 2012). For example, commercial, residential, industrial, agricultural and recreationally zoned lands may be correlated to the mobility patterns of urban occupants (Yuan and Raubal 2012). As historical Bluetooth data accumulates over time, researchers will be able to analyze time series movement data to predict the impacts of changing land use for policy makers. Bluetooth data can also be used for identifying unusually high occurrences of Bluetooth devices in a given area. These thresholds can be validated against mobility pattern, hot spot clustering, and urban rhythm research that has been previously accomplished using mobile phone data ( $\mathrm{Lu}$ 2000, Herrera et al. 2010, Yuan, Raubal and Liu 2012).

- Analyzing individual travel behavior: Bluetooth data can be used to explain the behaviors of individual residents within an urban environment. Previous studies used Bluetooth data to identify unique frequency patterns of repeated trips to classify users as infrequent, frequent, or very frequent travelers (Crawford, et al. 2018).

In summary, as advancing technology continues to improve the penetration rate of sensed devices, the accuracy and significance of Bluetooth data enabled research will continue to grow. However, the use of such Bluetooth datasets is rather limited, especially in understanding the functionalities of urban regions and land use patterns. This study tests the effectiveness of Bluetooth data in understanding urban dynamics on a case study in the City of Austin, Texas (ATX). This research has both practical and academic benefits. The practical benefit is, by analyzing urban patterns from Bluetooth data, we provide the ATX policy makers a practical framework to understand the dynamics of Austin's residents. The academic benefit is, this study validates new concepts such as "human sensing" and "social sensing" (Liu et al. 2015, Soliman et al. 2017, Doran et al. 2016) in the field of spatial sciences, which introduces new opportunities to monitor the human aspects of social life.

\section{Methodology}

Austin is a vibrant city that hosts many tourists from regional, national, and international destinations. It is essential for urban planners and policy makers to monitor and identify outlier mobility patterns during holidays and/or special events. The Bluetooth data used in this research are provided by the ATX Department of Transportation. The Bluetooth data was collected from January 2016 to August 2016 and totals over 81.6 million records with each record consisting of a time stamp when a device was read, a unique identifier, a location of the recorder, and a randomized unique MAC address tied to a Bluetooth device.

Because it is essential for urban planners and policy makers to monitor and identify outlier mobility patterns during special events, the research question here is how we can identify outlier mobility patterns from Bluetooth data. We analyze the 24-hour time series of unique Bluetooth devices for two pairs of time periods:

(1) May 28 - 30, 2016 (Memorial Day long weekend, during which in-state residents often take short trips) and May 7, 8, 14, 15, 21, 22 (six regular weekend days in May).

(2) March $21-25,2016$ (South by Southwest, also known as SXSW - an annual festival of film, interactive media, and music that attracts participants from all over the country) and March 7 - 11, 2016 (five regular week days in March).

Our goal is to identify the districts in Austin with the least and most similar patterns during these two pairs of time periods. We first aggregate the number of unique Bluetooth devices by hour for each Bluetooth sensor (Figure 1), so each sensor location is associated with a 24hour time series showing the temporal pattern of Bluetooth devices recorded. We then use a dynamic time warping (DTW) algorithm to measure the similarity of hourly mobility patterns between censors/locations. DTW has proven to be robust against distortion in time series (Zhang et al. 2008, Senin 2008) so it allows us to group similar patterns and identify outliers. Based on the DTW algorithm, we calculate the similarity of time series for the study periods for each Bluetooth location.

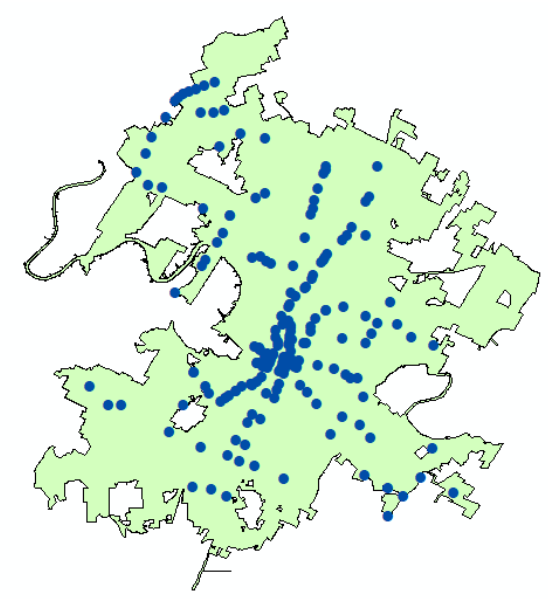

Figure 1. Bluetooth sensors in Austin.

\section{Results}

\subsection{Comparison between the Memorial Day weekend and regular weekend day patterns}

Figure 2 demonstrates the area in Austin with the least similar mobility dynamics (i.e., the intersection of Congress Avenue and Riverside Drive) between the six regular weekends and the Memorial Day long weekend. This is a downtown location with many hotels and tourist 
sites, such as the Town Lake Metropolitan Park and the Austin Convention Center. Figure 2a shows that this area is more active during night hours. The discrepancy of time series patterns between the two study periods is possibly due to the increased tourist activities during the Memorial Day long weekend. As a comparison, Figure 3 shows the area with the most similar patterns (i.e., the intersection of Lamar Boulevard and Parmer Lane) between the long weekend and regular weekends. This is a traditional residential area and is likely to be less impacted by any tourist activities during the holiday. The results indicate a correlation between urban land use and the mobility patterns reflected by Bluetooth data.

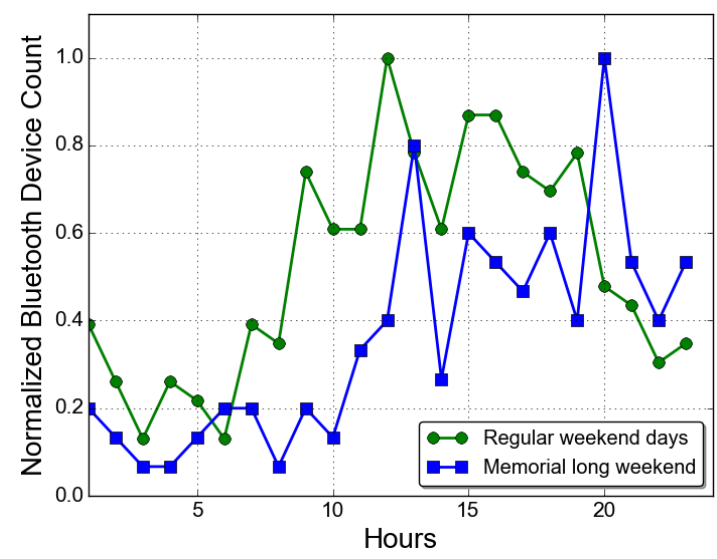

(a)

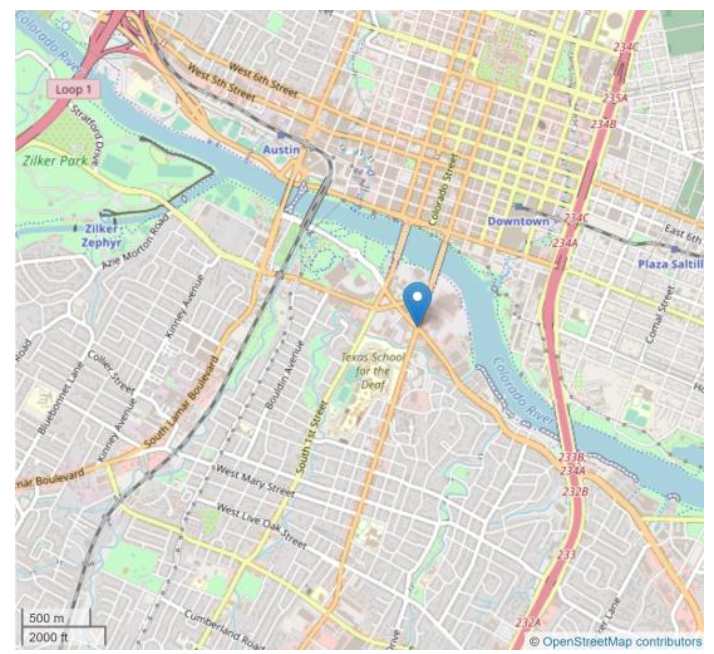

(b)

Figure 2. Dynamic patterns of the Congress Avenue/Riverside Drive intersection (a) time series of normalized Bluetooth device count; (b) base map from OpenStreetMap.

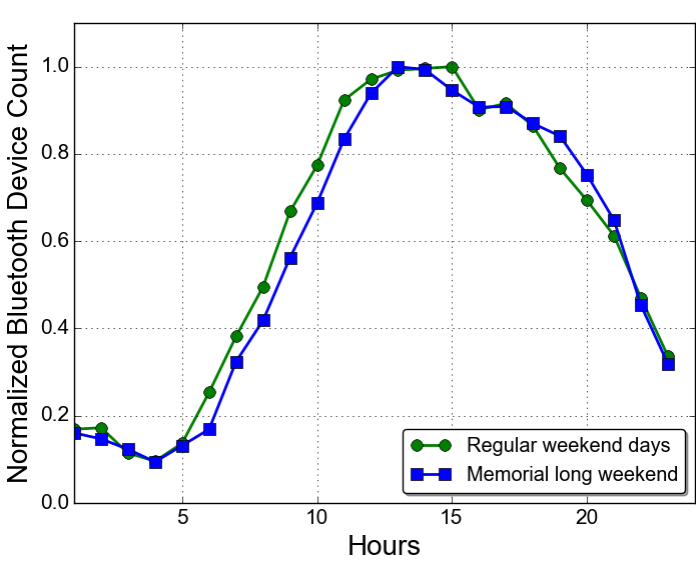

(a)

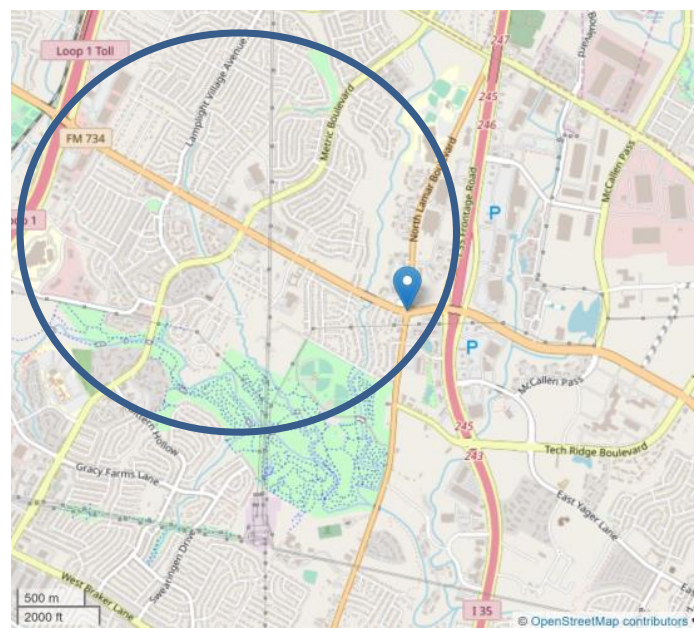

(b)

Figure 3. Dynamic patterns of the Lamar Boulevard and Parmer Lane intersection (a) time series of normalized Bluetooth device count; (b) base map from OpenStreetMap (circle shows a large residential subdivision).

\subsection{Comparison between the SXSW and regular weekday patterns}

Similarly, Figure 4 demonstrates the area in Austin with the least similar mobility dynamics between SXSW and a regular week in March. This is the intersection of Martin Luther King Jr. Boulevard and Lavaca Street. As can be seen from Figure $4 \mathrm{~b}$, this interaction is next to the University of Texas (UT) campus, where the mobility patterns during the SXSW week is very similar to that of a regular week. In addition, the intersection with the second highest similarity between the SXSW week and the regular week in March is Guadalupe Street and $21^{\text {st }}$ Street, which is also next the UT campus (Figure 5). This is potentially because universities have regular weekly schedules during the semester; therefore, students who live close to campus may follow similar activity patterns that are less impacted by special events like SXSW. 


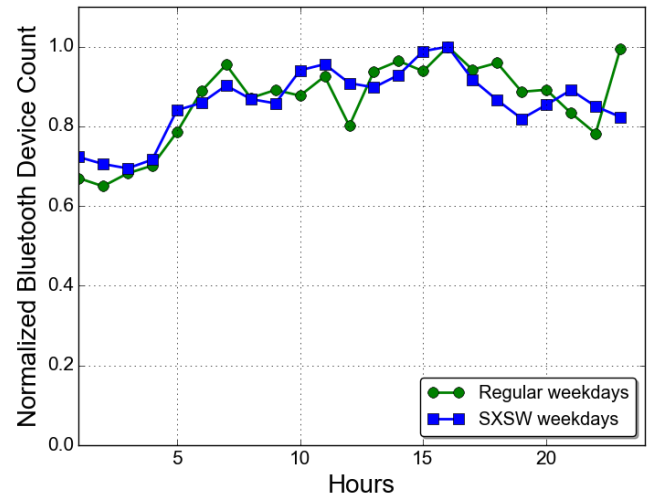

(a)

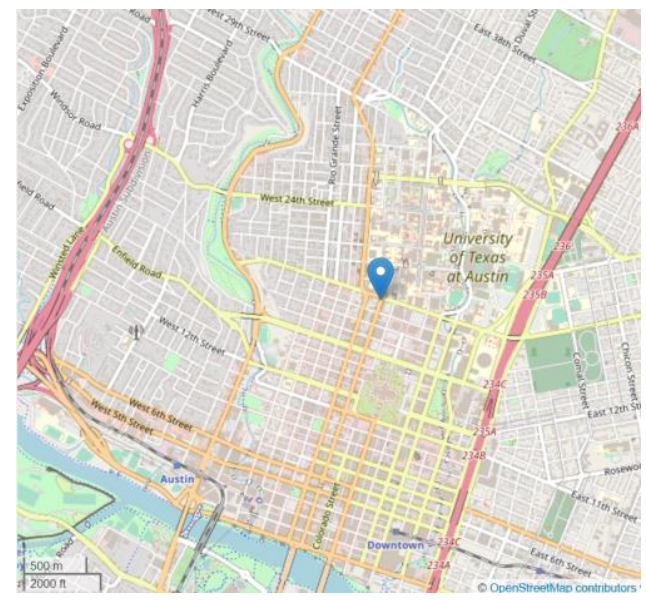

(b)

Figure 4. Dynamic patterns of Martin Luther King Jr. Boulevard and Lavaca Street (a) time series of normalized Bluetooth device count; (b) base map from OpenStreetMap.

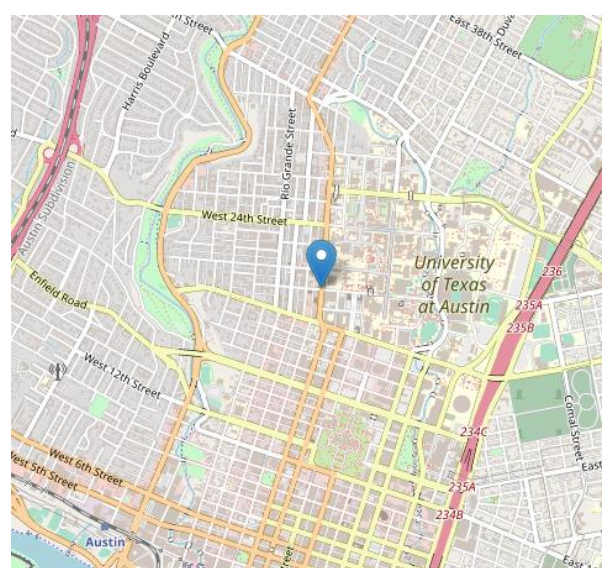

Figure 5. Dynamic patterns of the Guadalupe Street and $21^{\text {st }}$ Street.

On the other hand, the interaction between Lamar Boulevard and Martin Luther King Jr. Boulevard shows a very different hourly mobility pattern during the SXSW week (Figure 6a), where the mobility level during night hours is substantially higher during SXSW than regular weekdays. As can be seen from the base map (Figure 6b), this location is next to a city park (the "Pease District Park"); therefore, the increasing mobility is potentially due to the rise of night hour leisure activities at this location during SXSW. The results further confirm the findings in Section 4.1, where Bluetooth data effectively captured fine-grained urban dynamics and land use patterns.

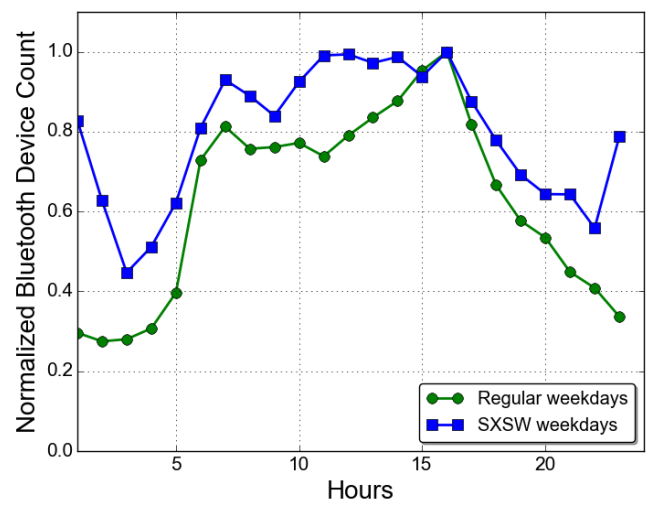

(a)

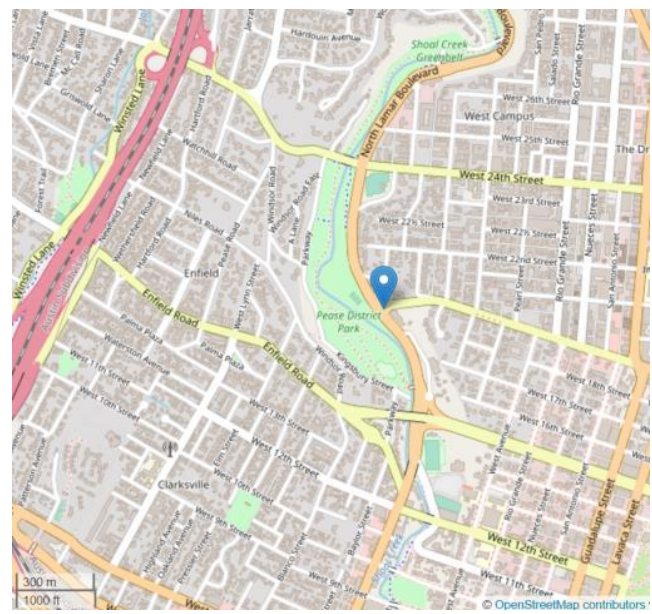

(b)

Figure 6. Dynamic patterns of the Lamar Boulevard and Martin Luther King Jr. Boulevard (a) time series of normalized Bluetooth device count; (b) base map from OpenStreetMap.

\section{Conclusion}

As shown in the results, Bluetooth data is effective in capturing urban dynamic patterns in both spatial and temporal dimensions. Compared to traditional land-use classifications (e.g., home, work), it is more useful in identifying finer-scale outlier areas and their temporal signatures. Our next step is to construct a distance matrix for the time series associated with each Bluetooth sensor and then conduct a hierarchical clustering analysis to identify clusters of urban functional regions. Because Austin hosts many regional and national festivals and events, we will also examine the time signatures during other special events, such as the Austin City Limits another annual festival of interactive media and music that attracts participants from all over the country. This research will benefit Austin residents and urban managers by generating a research framework, field-testing a statistical mechanism for analyzing urban dynamics from Bluetooth data, and identifying target areas for future ATX initiatives in urban planning. 


\section{References}

Ahas, R., A. Aasa, Y. Yuan, M. Raubal, Z. Smoreda, Y. Liu, C. Ziemlicki, M. Tiru \& M. Zook (2015) Everyday Space-Time Geographies: Using Mobile Phone-Based Sensor Data to Monitor Urban Activity in Harbin, Paris, and Tallinn. International Journal of Geographical Information Science, 1-23.

Anderson, J. M., N. Kalra, K. D. Stanley, P. Sorensen, C. Samaras, O. A. Oluwatola, Rand Corporation \& Rand Transportation Space and Technology (Program). 2014. Autonomous Vehicle Technology : A Guide for Policymakers. Santa Monica, CA: Rand Corporation.

Araghi, B. N., J. Hammershøj Olesen, R. Krishnan, L. Tørholm Christensen \& H. Lahrmann (2015) Reliability of Bluetooth Technology for Travel Time Estimation. Journal of Intelligent Transportation Systems, 19(3), 240-255.

Calabrese, F., L. Ferrari \& V. D. Blondel (2015) Urban Sensing Using Mobile Phone Network Data: A Survey of Research. Acm Computing Surveys, 47(2).

Chen, B. Y., H. Yuan, Q. Li, S.-L. Shaw, W. H. K. Lam \& X. Chen (2016) Spatiotemporal Data Model for Network Time Geographic Analysis in the Era of Big Data. International Journal of Geographical Information Science, 30(6), 1041-1071.

Costa, D. G., C. Duran-Faundez, D. C. Andrade, J. B. Rocha \& J. P. J. Peixoto (2018) Twittersensing: An Event-Based Approach for Wireless Sensor Networks Optimization Exploiting Social Media in Smart City Applications. Sensors, 18(4).

Crawford, F., D. P. Watling \& R. D. Connors (2018) Identifying Road User Classes Based on Repeated Trip Behaviour Using Bluetooth Data. Transportation Research Part A: Policy and Practice, 113, 55-74.

Delafontaine, M., M. Versichele, T. Neutens \& N. Van de Weghe (2012) Analysing Spatiotemporal Sequences in Bluetooth Tracking Data. Applied Geography, 34, 659668.

Doran, D., K. Severin, S. Gokhale \& A. Dagnino (2016) Social Media Enabled Human Sensing for Smart Cities. AI Communications, 29(1), 57-75.

Du, R., C. Chen, B. Yang, N. Lu, X. Guan \& X. Shen (2015) Effective Urban Traffic Monitoring by Vehicular Sensor Networks. IEEE Transactions on Vehicular Technology, 64(1), 273-286.

Filgueiras, J., R. J. F. Rossetti, Z. Kokkinogenis, M. Ferreira, C. Olaverri-Monreal, M. Paiva, J. M. R. S. Tavares \& J. Gabriel. 2014. Sensing Bluetooth Mobility Data: Potentials and Applications. In Computer-Based Modelling and Optimization in Transportation, eds. J. F. de Sousa \& R. Rossi, 419-431. Cham: Springer International Publishing.

Friesen, M. R. \& R. D. McLeod (2015) Bluetooth in Intelligent Transportation Systems: A Survey. International Journal of Intelligent Transportation Systems Research, 13(3), 143-153.

Herrera, J. C., D. B. Work, R. Herring, X. G. Ban, Q. Jacobson \& A. M. Bayen (2010) Evaluation of Traffic Data Obtained Via Gps-Enabled Mobile Phones: The
Mobile Century Field Experiment. Transportation Research Part C-Emerging Technologies, 18(4), 568583.

Liu, Y., X. Liu, S. Gao, L. Gong, C. Kang, Y. Zhi, G. Chi \& L. Shi (2015) Social Sensing: A New Approach to Understanding Our Socioeconomic Environments. Annals of the Association of American Geographers, 105(3), 512-530.

Lu, Y. 2000. Spatial Cluster Analysis of Point Data: Location Quotients Versus Kernel Density. In 2000 University Consortium of Geographic Information Science (UCGIS) Summer Assembly Graduate Papers. Portland, Oregon.

Malinovskiy, Y., N. Saunier \& Y. Wang (2012) Analysis of Pedestrian Travel with Static Bluetooth Sensors. Transportation Research Record, 2299(1), 137-149.

Poorthuis, A. \& M. Zook (2017) Making Big Data Small: Strategies to Expand Urban and Geographical Research Using Social Media. Journal of Urban Technology, 24(4), 115-135.

Reed, C. 2014. Bluetooth. Vero Beach, FL: Rourke Educational Media.

Salganik, M. J. 2018. Bit by Bit : Social Research in the Digital Age. Princeton: Princeton University Press.

Senin, P. 2008. Dynamic Time Warping Algorithm Review. Information and Computer Science Department, University of Hawaii at Manoa.

Shi, W., A. Zhang, X. Zhou \& M. Zhang (2018) Challenges and Prospects of Uncertainties in Spatial Big Data Analytics. Annals of the American Association of Geographers, 108(6), 1513-1520.

Silva, I. M. P. \& A. J. C. Moreira (2012) Evaluation of Bluetooth Technology as a Sensor of Urban Mobility. 7th Iberian Conference on Information Systems and Technologies (Cisti 2012).

Soliman, A., K. Soltani, J. Yin, A. Padmanabhan \& S. Wang (2017) Social Sensing of Urban Land Use Based on Analysis of Twitter Users' Mobility Patterns. PLOS ONE, 12(7), e0181657.

Yang, Z., M. L. Franz, S. J. Zhu, J. Mahmoudi, A. Nasri \& L. Zhang (2018) Analysis of Washington, Dc Taxi Demand Using Gps and Land-Use Data. Journal of Transport Geography, 66, 35-44.

Yuan, Y. \& M. Raubal. 2012. Extracting Dynamic Urban Mobility Patterns from Mobile Phone Data. In Geographic Information Science - 7th International Conference, 354-367. Columbus, USA: Lecture Notes in Computer Science, Springer.

Yuan, Y. \& M. Raubal (2016) Analyzing the Distribution of Human Activity Space from Mobile Phone Usage: An Individual and Urban-Oriented Study. International Journal of Geographical Information Science, 30(8), 1594-1621.

Yuan, Y., M. Raubal \& Y. Liu (2012) Correlating Mobile Phone Usage and Travel Behavior - a Case Study of Harbin, China. Computers, Environment and Urban Systems, 36(2), 118-130.

Zhang, B., K. Yan, S. Jiang \& D. Wei;. 2008. Walking Stability Analysis by Age Based on Dynamic Time 
Warping. In IEEE International Conference on Computer and Information Technology, 2008. 8th. 